\title{
The Impact of Program Context on Motivational System Activation and Subsequent Effects on Processing a Fear Appeal
}

\author{
Robert F. Potter, Indiana University \\ Michael S. LaTour, University of Mississippi \\ Kathryn A. Braun-LaTour, University of lowa \\ Tom Reichert, University of Arizona
}

\begin{abstract}
This manuscript reports three experiments investigating the impact of television programming context on the processing of a fear-appeal message. This is done using a dualmotivation system theory conceptualizing emotion as arising from activation of the appetitive and/or aversive motivational systems. Results show that, as predicted, sad programming activates viewers' aversive motivational systems, whereas comedic programming activates their appetitive motivational systems. Furthermore, by activating these systems through programming context, we were able to predict both retrospective self-report and real-time physiological reactions to a persuasive message employing a fear-appeal strategy. Theoretical and practical implications are discussed, as are suggestions for future experiments using the dual-motivation approach.
\end{abstract}

There is little doubt that one of the main motivations for tuning in to most prime-time television is to be moved emotionally. Whether looking for a sitcom that makes you laugh out loud, a crime drama that entices you with its intrigue, or a movie that tugs at the heartstrings, viewers often make selections based on their desire to have an emotional experience through television programming. Advertisers should be concerned about this, of course, because the impact of such mood inducement by television content may result in cognitive and emotional effects that carry over to the processing of commercials in programming breaks. This paper describes three studies that add insight to a well-developed stream of advertising research on the generation of mood by the programming context preceding ads and the effect of such on persuasive-message processing (Bello, Pitts, and Etzel 1983; Murry and Dacin 1996; Shapiro, Maclnnis, and Park 2002), as well as insight into works on fear appeals as a persuasive strategy (LaTour and Rotfeld 1997; Witte, 1992). Each of these previous studies, however, conceptualizes emotional valence as being a bipolar construct anchored by positive and negative. Current work in the 
psychology of emotion suggests that emotional states are actually the result of a dual, independent motivational system within the human organism-one positive and another negative. The current study begins applying this conceptualization to the world of advertising with the hope of both advancing theory and impacting practice.

The dual-motivation theory, developed by Cacioppo (Cacioppo and Gardner 1999; Cacioppo, Gardner, and Berntson 1999), springs from dimensional emotion theory (Bradley 1994; Cacioppo and Gardner 1999), which specifies that emotions can be mapped along two primary dimensions: arousal and valence. In other words, any emotional state can be placed onto two-dimensional affective space with axes consisting of a valence continuum (from negative to positive) and an arousal continuum (from calm to aroused) (Bradley and P. Lang 2000; A. Lang 2000; Osgood, Suci, and Tannenbaum 1957). It is under such a conceptual understanding, in fact, that much of the work in the area of emotional response to advertising operates. One of the ways that Cacioppo's dual-system approach expands this conceptualization, however, is by stressing the functional relevance for human survival of such a dichotomy, and the experienced emotions generated from it. Dual-system theory states that human beings have evolved to possess two independent motivational systems: the appetitive and the aversive. The former is associated with positive valence and approach behaviors, which have been developed to move the human organism closer to good things (for example, food and possible mates). The aversive motivational system, on the other hand, is an evolved negative response to potentially harmful situations or objects. It is fairly easy to understand how two such motivational systems would be naturally selected for over the course of human history. Organisms that were able to find food and mates while avoiding predators were more likely to produce progeny. Those with less attuned appetitive and aversive systems, however, would be less likely to procreate.

To our knowledge, this dual-motivation approach has not been used by advertising researcherscertainly not as applied to the topic of mood induction by emotional television programming and the subsequent effects on processing advertising messages that follow such programming. With dual motivation theory being such a fruitful theoretical approach in areas such as cognitive psychology, health communications, and neuroscience, it is our opinion that such an omission in the advertising literature is detrimental to the goal of fully understanding how human beings' emotional processing impacts interactions with advertising material. In this paper, we begin applying the dual-motivation approach to the study of advertising by investigating the impact of appetitive and aversive motivational activation on viewers processing of a fear-appeal message. 


\section{Background}

For decades, emotion has been conceptualized as consisting of two primary factors: valence and arousal. Early work by Osgood, Suci, and Tannenbaum (1957) used factor analysis of the English language to discover that emotional words coalesced around a description of positivity/negativity and the extent to which this valence was experienced-in other words, how calm or arousing it was. P. Lang (1979) developed a bio informational theory of emotional processing, a key component of which was a two-dimensional emotional space, where any experienced emotion could be mapped along the dimensions of positivity/approach and negativity/avoidance. Theories such as these state that the valence dimension is explicitly bipolar; in other words, the more positive something is, the less negative it necessarily must be.

Later work by Cacioppo and his colleagues (Cacioppo and Gardner 1999; Cacioppo, Gardner, and Berntson 1999; Ito and Cacioppo 2000) posited that emotion is better understood as the activation of two separate (positive/approach and negative/ avoid) valence systems. Because the systems were distinct, they could each be independently and simultaneously activated. The result of this conceptualization is four possible activation states: (1) reciprocal, where activation in one system increases and activation in the other system decreases; (2) uncoupled, in which activation in one system occurs while the other system remains unchanged; (3) coactive, which occurs when activation happens in identical directions across both systems; and (4) inactive, or a state in which neither positive nor negative valence systems are stimulated. Coactivation, which seems contrary to previous theories of emotional response, has been supported by work showing that people can feel both happy and sad simultaneously (Larsen, McGraw, and Cacioppo 2001).

Cacioppo has since combined this dual-motivation system approach with Miller's (1961) animal experiments investigating how novel surroundings are explored and conflict is avoid ed. In doing so, he produced descriptive activation functions of both the appetitive and aversive systems. These functions showed a steeper slope for the aversion response. Cacioppo termed this the negativity bias, claiming that the withdrawal system, when activated, responds much more quickly and with greater resultant arousal than the other system. The activation functions also exhibited a higher intercept for the appetitive system, which Cacioppo referred to as the positivity offset, suggesting that in a neutral environment, the human organism is naturally in an approach set that results in the exploration of unknown but neutral surroundings. 
Recently, A. Lang and her colleagues have begun using this dual-motivation system theory, and the concepts of negativity bias and positivity offset, as a promising approach through which to observe viewers' reactions to antidrug television public service announcements (PSAs) (A. Lang, Shin, and Lee 2005). In this paper, however, we attempt to build on that work by showing how a persuasive strategy used by advertising creators can interact with the programming context in which it is placed to impact the motivational systems of the intended audience. We predict that such an interaction will lead to effects not only in self-reported emotional response and behavioral intention, but also in real-time physiological response to the persuasive message.

\section{Experiment 1}

As an initial step, we were interested in determining whether the mood induced by sad and comic programming would activate the aversive and appetitive motivational systems, respectively. We conducted this experiment as a general test of Cacioppo's theoretical approach, for if one assumes that the human cognitive system is designed to generally respond to positive things in an approach mode while avoiding negative things, then arguably any genre of negative television programming should act as a prime for the aversive motivational system, and any genre of positive television programming should activate the appetitive system. However, much of the work in emotional response to television focuses on extreme ends of the valence continuum, with accompanying high emotional arousal-in other words, times when motivational systems are highly activated. For example, research has placed experimental participants in a positive state by showing them scenes of sexual situations ranging from innuendo to actual nudity and erotica (Gunter 2002; Jansen and Fridja 1994; Reichert 2002). Similarly, when trying to generate a negative emotional response, graphic fight scenes or news stories featuring bloody accident footage are common (Grabe et al. 2000; A. Lang, Newhagen, and Reeves 1996). On the other hand, very little research has been published employing more subtle stimuli to induce emotional activation through programming context, such as video that is only somewhat positive or somewhat negative and, although perhaps arousing to some extent, not likely to activate either the appetitive or aversive system to a great extent.

For our first experiment, therefore, we were interested in using these more understated forms of emotional entertainment to confirm that they, too, would activate the motivational systems described by Cacioppo. We designed a between-subjects experiment where roughly one-third of the subjects $(n=26)$ viewed comic fare preselected from popular movies, another third $(n=27)$ watched a sad scene selected from a popular theatrical movie release, and the last third $(n=26)$ watched a more 
neutral program. We hypothesized that the more positive program would activate viewers' appetitive motivational system, whereas the more negative program, though not visually graphic or terribly arousing, would still activate the aversive system to a measurable degree. We based this on an understanding of the cognitive system as comprised of associative memory networks that operate on the basis of spreading activation across nodes (Klimesch 1994). In other words, processing any piece of information activates memory structures not only for the single piece of information itself, but also for information associated with it in the individual's cognitive network. Operationally, therefore, placing subjects in a negative activation state by showing a sad film should stimulate memory networks associated with negative affect and the aversive motivational system. We hypothesized that such activation would subsequently cause viewers completing a word categorization task immediately after watching the sad television programming to respond faster to negative words than to positive words. Similarly, subjects who watched the comic programming would have positive memory networks stimulated within their cognitive system, and would therefore be placed in a slightly activated appetitive state. This would result in speeded response to categorizing positive words compared to negative words in a postexposure task. Finally, viewers watching affectively neutral television programming could be expected to have no activation associated with either the appetitive or the aversive systems. Specifically, then, our first hypothesis follows:

Hypothesis 1: Categorization speeds will be fastest for words congruent with the motivational state activated by the programming context and slowest for words that are incongruent to the programming context. Because no motivational activation is expected from neutral programming, word categorization speeds in that condition will be between those in other conditions regardless of word valence.

\section{Method}

Design

A one-factor design with three levels of emotional programming (slightly positive, neutral, and slightly negative) was employed. The levels were represented by three, 20-minute video segments prepared to induce activation of the motivational systems. The slightly positive stimulus was a comedy routine by Bill Cosby and a selection from the Jim Carrey movie Liar Liar. The neutral programming was a documentary about Albert Einstein. The slightly negative stimulus was a clip from the movie Steel Magnolias. These mood induction videos had been pretested to ensure the proper mood state (Filopowicz 2000; Puccinelli 1999). 


\section{Participants}

Seventy-nine undergraduates (50 female, 29 male) participated in this study for course credit. All provided informed consent prior to participating in accordance with Institutional Review Board guidelines at a large southeastern university.

Procedure

Participants were randomly assigned to one of the three conditions prior to their arrival at a computer lab. They were told that they would be completing some computer tasks after watching a videotape of television programming. They saw one of the three videos, depending on their assigned experimental condition, after which they were instructed on how to use the computer keyboard to indicate whether a series of words displayed to them individually on the screen were related or unrelated to the concept of fear. The words chosen to represent the aversive motivation construct were: fear, scared, frightened, tense, and anxious. The words selected to represent appetitive motivation activation were: happy, relaxed, calm, hope, and secure. The words were randomly presented. Half the participants were told to use the e key for identifying words associated with fear (i.e., the aversive words) and the $i$ key for words that were not associated with fear (i.e., the appetitive words); these keystroke instructions were reversed for the other half of the participants. The second trial had participants reverse response keys (the words were the same). The order of the response key trials was also randomly determined across participants.

\section{$\underline{\text { Results }}$}

We first looked at whether there was a handedness difference in the speed of response. There was not: Left-handed participants $(n=8)$ did not differ significantly from their right-handed counterparts, and thus the data were combined to address the hypothesis. We also tested whether the specific letter key used on the keyboard to categorize words as appetitive or aversive made a significant difference in speed of reaction time. It did not, and data from our two trials were therefore combined.

Our interest was in whether we could confirm activation of the aversive and appetitive motivational systems through a speeded categorization task following exposure to sad and comedic television programming, respectively. The hypothesis was based on the assumption that after participants watched a sad film their aversive system would be activated and, consequently, so would memory networks associated with the aversive concept of fear. Similarly, participants who watched a comic film would have their appetitive motivational systems activated and would respond more quickly 
to positively valenced words. Those exposed to a neutral film, on the other hand, would not have particularly strong activation in either system, and would therefore have moderate times in response to words of both valences.

We found there was a significant difference, $F(2,77)=3.79, p<.05$, and those in the aversively activated group were significantly faster in identifying the aversive-activation words ( $M=834 \mathrm{~ms}$ ) than those in the appetitively activated group ( $M=1,017 \mathrm{~ms}$ ) and those in the neutral group ( $M=933 \mathrm{~ms}$ ). Comparisons using the Tukey procedure found that only the groups that viewed negative and positive programming differed significantly from one another.

With the appetitive-activation words, the opposite was found. As predicted, those who watched the positive programming were faster ( $M=894 \mathrm{~ms}$ ) than those in the neutral group ( $M=961 \mathrm{~ms})$ and those in the negative programming group ( $M=1,074 \mathrm{~ms}$ )-a significant difference at $F(2,77)=4.13$, $\mathrm{p}<.05$-and Tukey post hoc tests found the positive group to be significantly faster than the negative group, but not the neutral group.

\section{$\underline{\text { Discussion }}$}

Our first hypothesis is supported: Watching a sad film does activate the aversive motivational system as indicated by speeded categorization of words associated with the aversive system, namely, fear-related words. Similarly, watching a light comedy does activate the appetitive motivational system. While the re action times of participants who saw neutral programming fell in between the times obtained for participants in the positive and negative-programming conditions for both the fear-related and non-fear-related words, these differences lacked statistical significance. However, informal and unrecorded debriefing of our participants resulted in reports that the neutral-programming condition was "boring" or "a waste of time." Certainly, either of these responses suggests a possible inadvertent activation of the aversive system in participants-which may explain the lack of significance in the data.

Although the primary theoretical justification for Experiment 1 was to confirm that subtly emotional programming would activate aversive and appetitive motivational systems-a central assumption for the independent variable manipulation in Experiments 2 and 3-the findings from the speeded categorization task should be of interest to advertising practitioners and call particularly for communication between creatives/copywriters and media planners. The words used in the categorization task could be considered typical on-screen graphic copy for everything from security systems and pain relievers (i.e., anxious, fear, tense) to financial services and hot tubs (i.e., secure, relaxed). However, the results suggest that the ability to cognitively recognize and semantically 
categorize individual words on a screen is affected by the activation of the audience's motivational systems as a result of the programming context in which the ad was placed. Participants who were appetitively activated by the comedy were more quickly able to cognitively respond to positive words and had more difficulty with negative words. The opposite was true for those who were aversively activated by the tearjerker movie. Being aware of the creative strategy and the phrasing of copy could therefore be used to guide the placement of ad campaigns within programming vehicles according to the type of activation expected from the programming and the words used in the commercial itself to attempt to achieve maximum cognitive activation in a target audience.

\section{Experiment 2}

With the results of Experiment 1 showing that even slightly emotional programming like comedy (subtly positive) and sadness (subtly negative) can activate what Cacioppo conceptualizes as the appetitive and aversive motivational systems, respectively, our next experiment explored the impact of such activation on the evaluation of a persuasive message. Specifically, we were interested in how activation of the motivational system would translate to the concept of audience arousal in response to a persuasive fear appeal (LaTour and Rotfeld 1997), an appeal that would, on its face, also activate the aversive system. In approaching the design of our second experiment, however, it was concluded that a neutral-programming condition was unnecessary. Because the speeded categorization task showed that the sad programming activated the aversive system and the humorous programming activated the appetitive system, using these two genres alone would be sufficient to investigate the impact of programming on the processing of subsequent fear appeals using Cacioppo's theoretical lens. To measure viewer arousal in Experiment 2, we relied on a series of research that conceptualizes arousal as a multidimensional construct consisting of positive "energy" arousal and the more negative "tension" arousal (Henthorne, LaTour, and Nataraajan 1993; LaTour 1990; LaTour and Pitts 1989; LaTour and Tanner 2003; Thayer 1967). According to Thayer (1989), tension arousal is associated with negative affective message tone and results in increased skin conductance in the palms of message recipients, along with the general constriction of muscle tissue. Thayer ties this tension reaction to the limbic system, and particularly the pituitary release of adrenocorticotropin (ACTH). It is suggested that the tension construct of arousal can be reduced with exercise, yoga, or other forms of meditation. The second dimension in Thayer's arousal concept-that of energy arousal-is associated with positive affective message tone and results in excitation of the Reticular Activation System (RAS) in recipients: As energy arousal increases, so does the release of adrenaline as a preparation for motor activity. 
Researchers in the area of marketing and advertising have successfully corroborated Thayer's notion that the two dimensions of the arousal construct-tension arousal and energy arousal-are empirically distinct in response to persuasive messages (see, e.g., Babin et al. 1998; LaTour 1990).

The similarities between Thayer's bi-dimensional arousal construct and Cacioppo's dual motivation theory should be apparent. In Experiment 2, the goal was to use the programming already identified with activation of the separate motivational systems (Experiment 1 ) as independent variables, and to test whether exposure to them affects self-report dependent measures designed to index Thayer's different dimensions of arousal, namely, the Activation-Deactivation Adjective Check (AD ACL; see Thayer 1989). The AD-ACL has a long history of use and validation (see LaTour and Rotfeld 1997) and features a series of five, four-point items for both the tension and energy arousal scales. The tension arousal scale includes such items as whether the respondent feels jittery, clutched up, and tense, while the energy scale includes whether the respondent feels energized, full of pep, and vigorous. The scale items are anchored by "definitely feel" and "definitely do not feel."

Generally speaking, the advertising literature points to a congruency effect" (Aylesworth and MacKenzie 1998; Gorn, Pham, and Sin 2001), where program affective context inducement carries over to attitude toward the ad. This is consistent with a dual-motivation approach, as the human cognitive system responds to mediated messages as if they were real (A. Lang 2006; Reeves and Nass 1996). In other words, when the mood of the programming is negative, the viewer responds as he or she would to negative things in real life, by activating the aversive system. This is particularly true when the topic of the mediated negativity "hits close to home" (Murry and Dacin 1996). When the mood of the programming is positive, however, the viewer reacts as if there were positive things in the real environment-by activating the appetitive motivational system. This differential activation is then a filter through which the processing of the persuasive message takes place. The affective tone of the advertisement becomes heightened or dampened depending on which motivational state the viewer is in.

In the second two experiments of the series, we placed a negatively toned PSA after the television programming. We predicted that the activation of the appetitive system occurring during the viewing of the comedy program would carry over to the processing of the PSA and result in higher levels of energy arousal for participants in that condition compared with those who watched the sad program. Likewise, aversive system activation resulting from watching the sad program would carry over to the processing of the negatively toned persuasive message and result in higher levels of tension arousal compared with those who watched the humorous programming. Therefore: 
Hypothesis 2: When placed in a negative-programming context, a fear appeal message will cause significantly higher levels of tension arousal than the same message placed in a positive-programming context.

Hypothesis 3: When placed in a positive-programming context, a fear-appeal message will cause significantly higher levels of energy arousal than the same fear appeal placed in a negative programming context.

The next hypothesis is based on the survival function of the dual-motivation systems. Dualmotivation theory posits that these systems have been naturally selected for because they result in increased success in keeping the human organism alive. This survival benefit is due to activation of the appetitive system being associated with moving toward the external stimulus that caused the activation; activation of the aversive system, on the other hand, results in active avoidance of the external stimulus. This approach/avoid dichotomy can be transferred to the concept of attitude toward the ad. If a viewer is placed in an aversive/avoid motivational set by television programming, his or her negative attitude toward the ad is expected to be exacerbated, whereas if the appetitive/approach motivational set is activated by the television programming, more positive attitude toward the ad is expected to be measurable. Therefore:

Hypothesis 4: A fear appeal placed in a negative-programming context will result in a significantly less positive attitude toward the ad than the same fear appeal placed in a positive-programming context.

When considering the impact of motivational system activation by television programming on behavioral intention following a PSA, logical arguments can be made for different expected outcomes by looking at the question according to different theoretical approaches. Stemming from past advertising research on congruency effects (Aylesworth and MacKenzie 1998; Gorn, Pham, and Sin 2001), one would predict that the positive mood experienced by those viewing the comic television programming would result in more positive attitudes toward the ad (as hypothesized above in Hypothesis 4), which, in turn, would result in a stronger likelihood to follow the suggestions made by the fear-appeal PSA. On the other hand, using a dual-motivation activation approach, an argument could also be made that the activation of the aversive system as a result of the negative television programming would be exacerbated by the negativity of the fear appeal (again, as hypothesized in Hypothesis 4). This increase in severity of aversive affect could be expected to lead viewers to want to avoid the negativity associated with the topic of the PSA. As a result, participants in this condition could be hypothesized to report greater intentions to follow the PSA's recommendations. 
Because the two arguments have both been validated by a string of published research, our final area of interest for Experiment 2 is investigated through the following research question:

Research Question 1: What will be the impact of motivational system activation through television programming on behavioral intention following exposure to a fear-appeal message?

\section{Method}

Design

This experiment again used a single-factor between-subjects design, with two levels representing positive and negative television-programming context.

Participants

Experiment 2 was conducted at the same southeastern university as the first experiment, with no duplicated participants. All participants $(n=103)$ provided informed consent, and were told that their responses would be anonymous and that there was no right or wrong answer on the questionnaire they would fill out.

Stimuli

The same sad and comedic programming used in the first experiment was used in the current one to activate the corresponding motivational systems. Immediately following each segment of programming, participants were exposed to the PSA. The customized fear appeal consisted of a modified version of an Australian "safe driving" PSA. The message featured two young couples driving on a country road. The male driver and his date were talking romantically when the two leaned over for a kiss. At that point, they realized they were driving toward the headlights of an oncoming truck. They screamed, swerved off of the road, and their vehicle rolled over several times. On coming to a stop, the camera showed that the female passenger in the front seat was dead-although her eyes remained wide open and blood ran down her face. The three other passengers presumably survived the crash, but could be heard groaning. At that point, the vehicle exploded in flame and passengers were heard screaming in guttural terror and agony as they burned alive. The screen turned black with a brief printed message stating, "Stay Alive-Keep your eyes on the road."

Because the PSA did not originate in the United States, there were concerns about its dialogue being comprehended by study participants. Therefore, a pretest was conducted, and these concerns 
were confirmed; participants had problems understanding the audio portion of the ad due to the heavy Australian accents of the actors. Consequently, the audio portion was reproduced using American voices. Furthermore, to ensure aversive activation, additional screaming was added during the scene where the car explodes into flames.

\section{Procedure}

Prior to their arrival at the research lab, participants were randomly assigned to one of two treatment groups according to the emotional programming they would be exposed to before the fear appeal. On arrival, participants were shown to their respective viewing rooms (i.e., participants were exposed in groups to the programming-with one room for the negative programming and another for the positive programming), and were told to relax for a few minutes prior to the start of the research process. This allowed them to acclimate themselves to the research environment prior to the beginning of the affective programming. They were told not to talk to each other during the viewing session.

Participants watched the affective television programming, which faded to black and was then followed directly by the PSA in a manner consistent with an advertising break. Immediately after the persuasive message, participants completed the self-report measures. They were then debriefed according to Institutional Review Board guidelines, and were thanked and dismissed.

\section{Data Reduction}

Factor analysis supported the dimensionality of "tension" and "energy" arousal and the Cronbach $\alpha$ s were .80 and .78 , respectively, both well above the common benchmark of .70 . Several sixpoint items for attitude toward the ad, including "good," "appropriate," "high quality," "interesting," and "irritating" (reverse scored) were used, with respondents asked to rate the ad on each item using scales anchored by "Yes, definitely" and "No, definitely not." Cronbach's coefficient $\alpha$ for this scale was .74. Factor analysis also supported unidimensionality. Based on feedback from a preliminary focus group as to what type of outcome measure made the most sense, a single six-point item, "I intend to be more alert driving to night," anchored by "strongly agree" and "strongly disagree" was also included. While a single-item measure is somewhat of a limitation, following the initial focus group discussions focusing on the nature of the PSA and its intended effect, it was determined to clearly be the most logical fit with the experimental stimulus. 


\section{Results}

Hypothesis 2 predicted that a negatively toned PSA, in the form of a fear appeal, would result in significantly increased levels of tension arousal when placed in a congruent negative programming context than the same PSA placed in a positive context. This hypothesis was supported, with tension arousal significantly higher, $\mathrm{F}(1,101)=15.4, \mathrm{p}<.05$, in the aversively activated group compared to the appetitively activated group, $M=14.09$ versus $M=11.02$, respectively. In addition, in contrast to the findings of LaTour and Rotfeld (1997), self-reported tension and energy arousal were negatively correlated, $r=-.227, p<.05$.

Hypothesis 3 predicted that self-reported energy arousal following the PSA would be significantly greater in participants whose appetitive activation system had been activated by the comic programming than those who had been aversively activated by the sad programming. The statistical analysis returned a significant result, $F(1,101)=27.3, p<.05$, with mean analysis confirming the hypothesis, as participants who had viewed the comic programming had greater energy arousal ( $\mathrm{M}=$ $11.28)$ than those who had viewed the sad programming $(M=7.98)$. For Hypothesis 4 , we predicted that attitude toward the ad would be lower for those who had been aversively activated prior to exposure to the PSA than for those who had been appetitively activated. This hypothesis was confirmed, $F(1,101)=$ 4.4, $\mathrm{p}<.05$, with attitude toward the ad significantly less positive in the group of participants that had viewed the negative programming $(M=17.69)$ than those that had viewed the positive programming ( $M=19.82$ ).

Our final interest area in Experiment 2 was a research question that asked what the impact of motivational system activation would be on behavioral intention following the fear appeal. This was framed as a research question because of competing theories in the literature. Congruency effect literature would suggest that those who watched the comic programming would have transferred the positive affect to a greater likelihood of agreeing with the suggestions of the mood-congruent fear appeal. An argument could also be made from a motivational system activation standpoint, however, that if viewers watched negative programming and were then exposed to a negative fear appeal, the heightened activation of the aversive system would make the consequences described in the PSA seem even more dire. As a result, viewers in this condition would be expected to be more likely to comply with the suggestions made by the PSA.

Results suggest that while attitude toward the ad is more positive in the appetitive activation condition, behavioral intention to be more alert while driving is significantly stronger for those who saw 
the PSA following the negative programming, $M=4.30$, versus $M=3.70$ in the positive-programming condition; $F(1,101)=3.94, p<.05$. This would suggest an interpretation more in line with Cacioppo's motivational system activation model. Further support for this view is provided by a significant correlation between tension arousal and behavioral intention to be more careful, $r=.280, p<.05$.

\section{$\underline{\text { Discussion }}$}

This experiment was designed to examine the dual-motivation approach to emotional experience during a negatively emotional PSA given unique programming context situations. All three predictions were made according to Cacioppo's conceptualization of experienced emotion as stemming from the activation of two independent motivational systems-and each were supported. Furthermore, a research question inquired whether results focusing on behavioral intention surrounding the focal topic of the PSA would support the advertising congruency literature or Cacioppo's theory. Once again, a dualmotivation approach seemed to be the best explanation for the data obtained, although interpretation of this finding should be done with caution due to the use of a single-item measurement of the intention construct.

Results also show that the same persuasive message can cause activation of different arousal constructs depending on respective motivational systems activated via different programming contexts in which the persuasive message is placed-a finding that, once again, suggests the importance of practitioners understanding the emotional tone of their campaign along with the likely programming context into which it will be placed.

It is interesting to note that the dependent variables used in Experiment 2 were, in fact, designed to assess two dimensions of experienced arousal under a different theoretical rubric (Thayer 1989). We associated Thayer's tension arousal and energy arousal constructs with activation of what Cacioppo refers to as the aversive and appetitive motivational systems, respectively. This association was supported by the data.

Of course, the impact of persuasive messages occurs in real time. The dependent variables employed in Experiment 2 were retrospective self-report measures and therefore may mask differences between conditions that occur during the on-line processing of the fear appeal. To test for these differences, a third experiment was conducted. 


\section{Experiment 3}

Results from our first two experiments suggest that even slightly emotional television programming activates the corresponding motivational systems in viewers. Furthermore, such activation has an impact on the post hoc evaluation of a persuasive message using a fear-appeal strategy. In the final experiment, interest turned to examining how activation of the aversive or appetitive motivational systems affected the real-time emotional response to the PSA using psychophysiological measures.

Results from Experiment 2-where the impact of motivational system activation was measured using Thayer's AD-ACL-provide guidance as to which psychophysiological indices would be most applicable to assess this on-line impact. If viewers were first exposed to negative television programming, their tension arousal levels were significantly greater when assessing the fear-appeal PSA compared with viewers who were first exposed to the comic television programming. Thayer identifies tension arousal with increased sweating of the palms and muscular tension, and it was therefore decided to use skin conductance and electromyographic (EMG) activity of the corrugator, or frown, muscle groups in the face as dependent variables in Experiment 3.

Measures of eccrine sweat gland activity in the hands have long been recognized as an indicator of emotional activity (Dawson, Schell, and Fillion 2000; Stern, Ray, and Quigley 2001). Unlike other sweat glands, which excrete in response to temperature increases, the eccrine glands are under the control of the autonomic nervous system and provide a physiological correlate for increases in experienced arousal in the human organism. Although more intrusive than paper and pencil measures, skin conductance provides a way of mapping in real time the arousal experienced during entertainment programming in media (A. Lang 2000; A. Langet al. 1999; Potter and Choi in press). Furthermore, researchers looking at advertising specifically have found it useful as a way of indexing arousal of the sympathetic system (Bolls and Potter 1998; Hopkins and Fletcher 1994; A. Lang et al. 2003).

Under Cacioppo's theoretical rubric, physiological arousal is indicative of motivational system activation--either appetitive or aversive. Results of Experiments 1 and 2 both suggest that a subtly negative programming context activated the aversive motivational system, whereas slightly positive programming activated the appetitive system. If this is the case, arousal levels would be somewhat elevated in both conditions, prior to viewing the negative PSA. The question then focuses on the expected behavior of skin conductance (as an indicator of motivational system activation) as the cognitive task shifts from processing the programming context to processing the negative PSA immediately following it. 
In the negative-programming condition, the message on the screen changes from a sad movie to the similarly negative fear appeal message; the tone of the persuasive message further activates the viewer's aversive motivational system. This increase in activation should be observable as an amplification in skin conductance activity as the fear builds to the culmination of the PSA where the car explodes and the occupants scream. For participants in the positive-programming condition, however, it is the appetitive motivational system that is initially activated. When this is replaced by the fear-appeal message, the change in motivational relevance leads to a simultaneous deactivation of the appetitive system and activation of the aversive system. The deactivation of the appetitive system is expected to lead to a decrease in skin conductance level (SCL). And although one might suspect that this decrease in physiological arousal would be replaced by the activation of the aversive system as a result of the fear appeal, research using the startle probe paradigm shows that the presence of appetitive activation due to a positive foreground context results in initial inhibition of the aversive activation response (Bradley, Cuthbert, and P. Lang 1991). In other words, the initial appetitive activation would prevent the immediate "ramping up" response by the aversive system. This, combined with the fact that the arousal associated with the appetitive activation would be decreasing at the same time, leads to the following prediction:

Hypothesis 5: When placed in a negative-programming context, the increased activation of the aversive motivational system by a negative PSA will result in greater skin conductance activity compared with the same PSA placed in a positive-programming context.

Similar predictions can be made regarding the physiological index of emotional valence-facial EMG. Measuring facial EMG is the process of quantifying the summative amplitude of electrical firings by a particular skeletal muscle group (Stern, Ray, and Quigley 2001). Darwin (1872) was one of the first to suggest that a basic goal of much muscular activity is the communication of an organism's emotional state. It is through the muscles in the face, particularly, that emotional valence is communicated using the zygomatic (smile) and corrugator (frown) muscle groupings (Tassinary and Cacioppo 2000; Ekman 1993). Psychophysiologists have validated the measurement of smile and frown muscles in the face using a variety of stimuli such as still picture slides (P. Lang et al. 1993), environmental sounds (Jancke et al. 1996), positive and negative spoken words (Wexler et al. 1992), and auditory tones of various intensities (Dimburg 1990). While not as common a measure of physiological responses to media programming, several recent studies have shown that facial EMG can be valuable for capturing the real- 
time reactions of target audiences as they respond to advertising (Bolls, A. Lang, and Potter 2001; Hazlett and Hazlett 1999; Lee and Potter 2005).

Our experiments focused on the degree of negative affect generated by a negatively toned fearappeal PSA and how that is impacted by the emotional tone of the programming preceding it. For that reason, our prediction focuses on corrugator (frown) muscle activity during the viewing of the PSA. During the viewing of the negative programming, the aversive motivational system was activated, and we would therefore expect that the corrugator muscle group would be activated as well. When the programming context was replaced by the fear appeal, the aversive activation would continue, and arguably increase, as the subject matter of the PSA was (by design) even more highly negative than the moderately negative sad film. Guided by the work of Larsen, Norris, and Cacioppo (2003), we expected that the corrugator muscles would be even less active during the viewing of the comedic programming than during neutral baseline conditions as a result of the appetitively activated state. Therefore, similar to the skin conductance predictions, we expected that this initial appetitive activation would prevent the immediate "ramping up" response by the aversive system with the processing of the fear appeal. Therefore, we propose our final hypotheses:

Hypothesis 6a: There will be a main effect of programming content of corrugator activity during PSA processing, with greater activity following negative programming compared with positive programming.

Hypothesis 6b: There will be an interaction between programming context and time on corrugator activity, such that muscle activity during PSA processing will increase more quickly for participants following negative programming compared with positive programming.

Using the same design and stimuli as Experiment 2, 46 participants completed this procedure individually in exchange for credit in courses offered at a different southeastern university than Experiments 1 and 2. All participants provided informed consent prior to completing the procedures and could withdraw from participation at any time (none withdrew, however).

After arriving at the lab for their scheduled appointment, participants were greeted by the researcher and told that they would be completing two experiments designed to investigate how people process television messages. The first study explored how people process different types of television news. The stimuli for that study contained no advertisements or PSAs, and the data are not reported here. Participants were also told that while they were watching the television messages, researchers would be recording how their body responds by using sensors placed on their arms, hands, and above their eyebrows. 
After providing written consent, physiological electrodes were attached to the participant. The participant's palm was uniformly hydrated with distilled water and two standard-size AG/AGCL electrodes containing electrically neutral gel were placed on the participant's nonwriting hand to measure skin conductance. Two mini AG/AGCL electrodes containing conductive gel were placed over the corrugator muscle group above the left eyebrow, after removing dead skin cells with a cotton pad containing rubbing alcohol and pumice. Three standard electrodes were also placed on the participant's arms to collect heart-rate data for the television news study. Because cardiac activity was not a part of our hypotheses in the current experiment, no information about cardiac data collection, cleaning, reduction, or analysis is presented here.

The participant first completed the stimuli-presentation phase of the television news study, which consisted of watching nine news stories and completing a questionnaire after each one. The participant was then instructed to watch a portion of a television movie (the motivation-activation stimulus for the current study) and told that he or she would be asked questions afterward. An equal number of participants $(n=23)$ were shown the positive and negative programming prior to the PSA. Physiology data were collected during the final 5 minutes of the programming context and during the entire 50 seconds of the PSA. The participant then completed a short series of memory tests for the television news study, was debriefed according to Institutional Review Board guidelines, and was thanked and dismissed.

\section{Physiology Data Collection, Editing, and Reduction}

Both the skin conductance and EMG data were collected as analog signals sampled 20 times per second using a Coulbourn skin conductance coupler and bioamplifier, respectively. The EMG data were also passed through a high- and low-pass filter, and then through a contour integrator, for signal smoothing.

Skin conductance and corrugator level activity was calculated and output on a second-by-second basis using the computer program VPMAnlog (Cook 2003). Change scores for both SCL and corrugator EMG level in each participant were computed for each second of the PSA by subtracting the level at the baseline second before the PSA started from the level at each second of the PSA itself. These were then aggregated into 5-second segments over the course of the 50-second PSA. 


\section{$\underline{\text { Results }}$}

Hypothesis 5 predicted that participants whose aversive motivational system had been activated by a negative television program would have greater autonomic arousal activity during the negative PSA than those who had been appetitively activated by a comic television program. To test this hypothesis, we analyzed both the number of spontaneous skin conductance responses (SCRs) that participants experienced, as well as their overall SCLs during their viewing of the PSA. Spontaneous SCRs are a way of describing short-term increases in arousal in response to nonspecified occurrences in the environment. They are momentary rises in level, usually having a predefined amplitude and rise time. For this study, we defined a skin conductance response as an increase of at least .10 microsiemens over, at most, a two-second time frame. Skin conductance level, on the other hand, is a measure used to gauge the overall level of arousal across a longer duration (Stern, Ray, and Quigley 2001).

Due to experimenter error or equipment malfunction, skin conductance data were lost for eight participants (four in each condition). We analyzed the obtained SCR data using three measurements common in psychophysiological research (Dawson, Schell, and Fillion 2000). First, we counted the frequency of SCRs. We documented the amplitude of the largest SCR, and calculated the mean amplitude during the PSA exposure. Dawson et al. (2000) report a low correlation between these three measures of SCR and suggest that each be analyzed separately as sources of independent information. Therefore, one-way ANOVAs were conducted on the three to determine whether there were statistical differences between the arousal responses of those who watched the positive programming compared with those who watched the negative programming.

We operationally defined $\mathrm{H} 5$ by predicting that all measures would be greater for participants in the negative-programming condition compared with those in the positive-programming condition. A small percentage of the population is electro dermally nonresponsive (i.e., exhibit no SCRs) and are often excluded from data analysis (Dawson, Schell, and Fillion 2000). Therefore, we analyzed data from all participants who exhibited at least one SCR during the PSA presentation ( $n=35)$. H5 was supported for the largest amplitude data, $F(1,33)=5.211, p<.05$, with those in the negative-programming condition having significantly greater largest amplitudes $(M=2.237, S D=1.691)$ compared to those in the positive-programming condition $(M=1.127, S D=1.152)$. The two other SCR measures failed to reach statistical significance.

The hypothesis also predicted that participants in the negative-programming condition would already have activation of the aversive motivational system, which would then be even further activated 
by the negative fear appeal. Those who saw the positive-programming context, however, would experience a switch in activation of the two motivational systems as a result of the transition from comedy (appetitive activation) to fear appeal (aversive activation). Operationally, we expected this to manifest itself in a dampening of SCL for those in the positive context and an exacerbation of SCL for those in the negative context. To test this, a $2 \times 10$ repeated-measures MANOVA (multivariate analysis of variance) was conducted comparing the change score values for each condition during each of the ten 5 -second segments of the PSA. This resulted in a significant main effect for condition, $F(1,36)=7.295$, $p<.05$. As can be seen in Figure 1, those who saw the negative programming and were aversively activated had significantly greater SCLs throughout the fear appeal than those who saw the positive programming. Across the entire duration of the PSA, the condition $x$ time interaction was not statistically significant $(F<1)$. However, as can be seen in Figure 1, although both groups had an increase in SCL at time segment 5 (when the car crash occurred in the PSA) and segment 9 (when the car exploded), this increase is exaggerated for those who had been previously exposed to negative programming. Looking only at the most marked difference between the two groups over time-that being the exaggerated reaction to the car accident by negative-context participants between time segments 5 to 9-the results of the $2 \times 5$ MANOVA show a significant condition $x$ time interaction in the quadratic trend, $F(1,36)=4.787, p<.05$.

We used similar reasoning when predicting the corrugator muscle differences between the two groups. Because participants in the negative-programming context had already been aversively activated, the subsequent processing of the negative fear-appeal PSA was expected to lead to greater corrugator activity for them than for participants in the positive-programming context, who, because they were appetitively activated, had to decrease the activation of that motivational system while simultaneously increasing their aversive activation associated with the processing of the PSA. This was tested statistically as a main effect for the change scores in corrugator activation level aggregated into 10 five-second segments. Due to equipment malfunction or experimenter error, corrugator data were unavailable for eight participants (final $\mathrm{n}$ for positive- and negative-programming context conditions were 18 and 20, respectively). The visual representation of these change scores can be seen in Figure 2. As predicted, the $2 \times 10$ repeated measures MANOVA resulted in a main effect for programming context, $F(1,36)=6.187, p<.05$, with those in the negative condition showing increased activity and those in the positive condition having their frown muscle activation attenuated. We also predicted an interaction between programming context and time on corrugator activity, expecting that muscle activity would increase more quickly for participants following negative programming compared with 
positive programming. The trend lines in Figure 2 show that, indeed, corrugator response in the aversively activated participants increased over time, whereas it decreased slightly over time (except during the initial car crash during segment 5-7) for those who were appetitively activated.

\section{$\underline{\text { Discussion }}$}

Results from Experiment 3 support and extend those of Experiment 2: Viewers placed in a negative activation state by the television program they were watching had an increase in tension arousal when exposed to a fear appeal. However, the physiological results illuminate how time plays a factor in audience response to a fear-appeal message depending on the programming context in which it is placed and the subsequent motivational system that is activated by such a context. Those participants whose aversive motivational systems had been activated by even a subtly negative programming context experienced not only a greater reflexive arousal reaction to the fear appeal, as indicated by significantly greater amplitude in the largest SCR, but as Figure 1 shows, this arousal reaction increased throughout the duration of the message. Meanwhile, participants whose appetitive motivational state was slightly activated by the comic program had low initial SCLs, which, although still responding to extremely emotional content in the fear appeal (i.e., the explosion of a car with passengers inside), remained dampened throughout the 50-second message as the appetitive motivational system became deactivated but still placed enough of a damper on the activation of the aversive system. Given such an explanation, in fact, the lack of significance in the overall frequency of SCRs is not surprising with inductive hindsight. Because the participants saw the exact fear appeal regardless of their programming context condition, the same content and structural features should have been eliciting SCRs across context condition. The change in activation from one motivational system to another was, however, dampening the range of response for those who were appetitively activated by the comedy programming.

This same general pattern can be seen in Figure 2, which shows that when aversive motivation is activated, corrugator muscles responded to a much greater extent over the course of the fear appeal when compared to processing the same message under a condition of appetitive activation. Once again, even though participants who have been appetitively activated can be shown to have corrugator response to highly negative situations in the fear appeal (between segments 4 and 5), having been placed in an approach mode by the comedy, corrugator activity never rises above programming context baseline. 


\section{FIGURE 1}

\section{Effect of Programming Context on Skin \\ Conductance Level (SCL)}

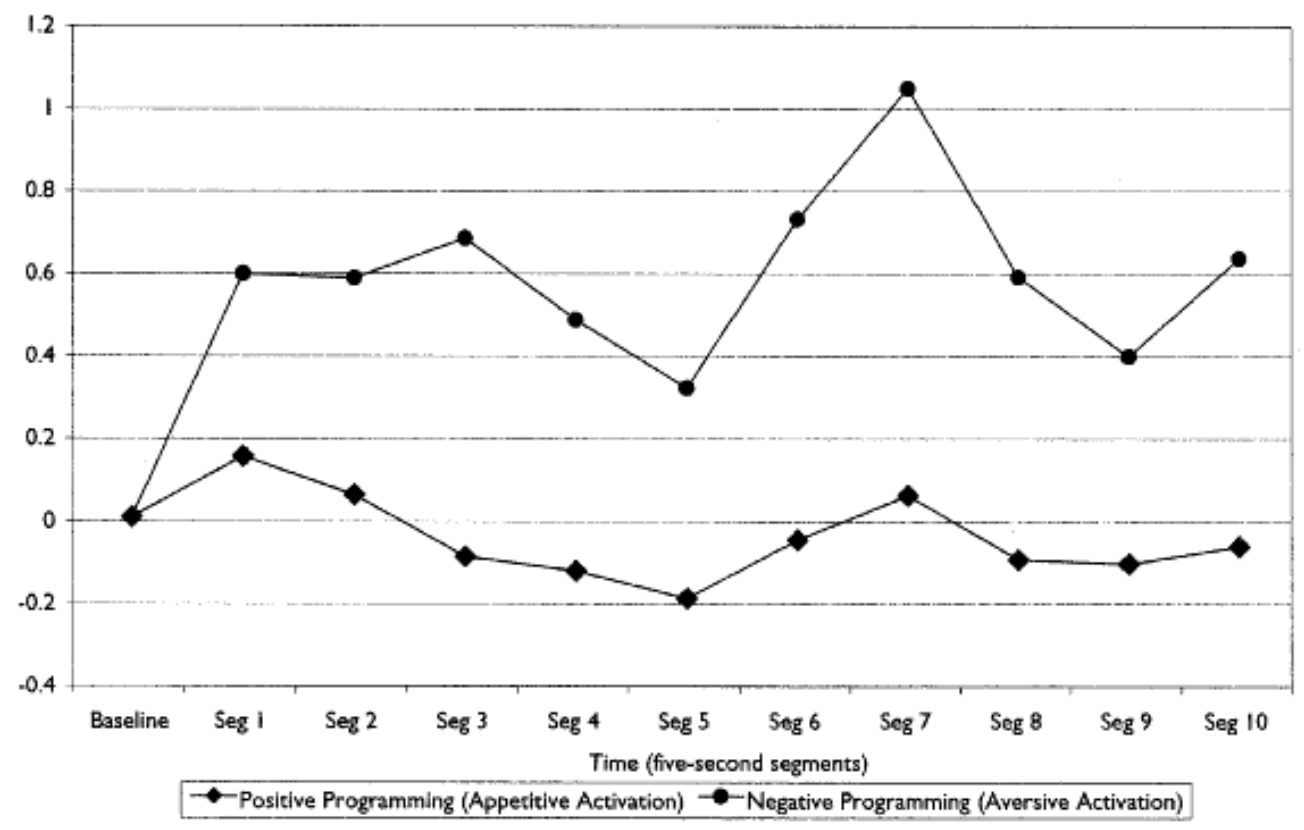

\section{FIGURE 2}

\section{Effect of Programming Context on}

Corrugator Muscle Activity

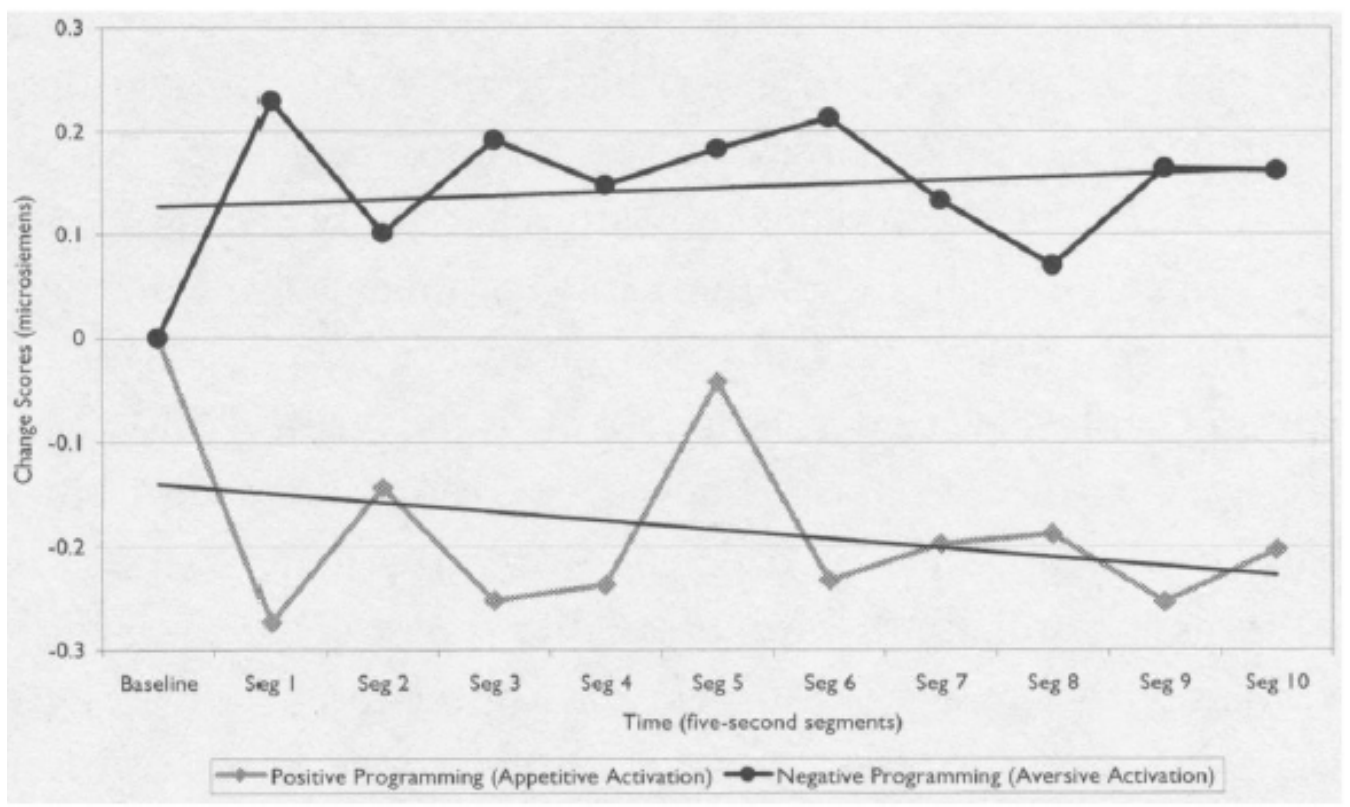




\section{General Discussion and Implications}

The primary goal of this manuscript was to explore how Cacioppo's dual-motivation activation model of emotion (Cacioppo and Gardner 1999; Cacioppo, Gardner, and Berntson 1999) could be used to investigate the impact of emotional television programming on the processing of a persuasive message. Although this model has been employed by others looking at persuasive messages (A. Lang, Shin, and Lee 2005), this is the first study we are aware of that uses it to explore not only how separate motivational systems in the human organism impact the processing of the persuasive message itself, but also how the programming occurring before the message activates the motivational systems and subsequently impacts advertisement processing. In a series of three experiments, Cacioppo's dualmotivation conceptualization led to seven confirmed hypotheses concerning the processing of a fearappeal message following emotional programming. Furthermore, data obtained in pursuit of a research question concerning behavioral intention subsequent to the negatively toned PSA supported the dualmotivation conceptualization more than "congruency effect" found in the advertising literature (Aylesworth and MacKenzie 1998; Gorn, Pham, and Sin 2001).

Theoretically, our results robustly support a dual-motivation model, and the strongest implication from our data applies to advertising researchers, who are urged to consider employing this theory in future work on advertising processing. Perhaps the greatest justification for employing this theory in advertising research is its basis in a biological understanding of human beings and their need to survive via consumption and the avoidance of harm. Satisfaction of these needs seems to be at the heart of most advertising campaigns, not just those that employ negatively valenced creative strategies such as fear appeals. Future research should attempt to replicate and extend these findings, searching for other ways to apply Cacioppo's conceptualization of two individually operating motivational systems to the processing of advertising messages.

To date, A. Lang and her colleagues have published interesting work in identifying primary motivational activation traits within individuals (A. Lang, Shin, and Lee 2005). Although the arousal level (i.e., activation) of both the positive and negative motivational systems is best viewed as a continuum, their early work has conceptualized the two individually responsive systems as resulting in four personality types: inactives are individuals with a generally low positivity offset and low negativity bias; risk takers are those with high positivity offset and low negativity bias; risk avoiders are those with low positivity offset and high negativity bias; and coactives are individuals whose appetitive and aversive systems are usually in a state of high activation. The implications of resting motivational activation 
states on the processing of persuasive messages should be investigated. Furthermore, as it is becoming more common for media buyers to use psychographic data as a supplement, or even a replacement, for basic sex/age demographic information (Mandese 2005), it is fascinating to consider how the availability of data such as the "expected, average motivational state" of viewers for certain programs could impact media-buying behavior, creative strategies, or expected return on investment. Although these data are not available at present, advertising scholars should explore how motivational personality types can help predict preference for specific media genres (i.e., sitcoms, episodic police procedurals, etc.)information that could then assist media buyers in considering the likelihood of certain motivational states in the audience of shows used to schedule persuasive messages.

In these experiments, the two motivational systems-appetitive/approach and aversive/avoidwere activated by a light comedy and a "tearjerker" movie, respectively. Results of all three experiments suggest that overly arousing material is not necessary to have an impact on the processing of subsequent persuasive messages. In fact, negative affect and increased behavioral intention were found in response to the negative PSA placed after television programming that activated the aversive system. This lends itself to several practical implications. For example, media buyers for social-marketing campaigns are advised to place fear appeals-or PSAs that generate high tension energy through aversive activation-in programming where even slightly negative content may be expected. Such programs might include sad or emotion-laden movies, daytime dramas, daytime talk shows (e.g., Montel, Maury Povich), and some news programs. For campaigns that depend on free air time or "pro bono" support, a list of recommended program types or genres should accompany the PSA.

Along the lines of creative strategy, it is possible that persuasive messages themselves could be designed to activate the appetitive system, the aversive system, or both. For example, the PSA tested in the present study could be labeled "fear rising" because the fear-evoking content builds toward the end of the spot, with resulting maximal activation of the aversive system. However, another pattern within the creative strategy of PSAs is one of "fear relief." In these messages, fear is evoked to grab attention during the first moments (i.e., highly activated aversion). The fear is then intermingled with factual and personal-efficacy information (coactivation; Witte 1992), and then finally replaced by a positive outcome (appetitive activation). The interaction between these creative strategies, the motivational traits of viewers, and the activation of motivational systems through programming context provides opportunities for many future studies. 


\section{References}

Aylesworth, Andrew B., and Scott B. MacKenzie (1998), "Context Is Key: The Effect of Program-Induced Mood on Thoughts About the Ad," Journal of Advertising, 27 (2), 17-31.

Babin, Barry J., William R. Darden, and Laurie A. Babin (1998), "Negative Emotions in Marketing Research: Affect or Artifact" Journal of Business Research, 42 (3), 271-285.

Bello, Daniel C, Robert E. Pitts, and Michael J. Etzel (1983), "The Communication Effects of Controversial Sexual Con tent in Television Programs and Commercials," Journal of Advertising, 12 (3), 32-42.

Bolls, Paul D., Annie Lang, and Robert F. Potter (2001), "The Effects of Message Valence and Listener Arousal on Attention, Memory, and Facial Muscular Responses to Radio Advertisements," Communication Research, 28 (5), 627-651.

, and Robert F. Potter (1998), "I Saw It on the Radio: The Effects of Imagery Evoking Radio Commercials on Listeners' Allocation of Attention and Attitude Toward the Ad," in Proceedings of the 1998 Conference of the American Academy of Advertising, Darrell D. Muehling, ed., Pullman, WA: American Academy of Advertising, 120-130.

Bradley, Margaret M. (1994), "Emotional Memory: A Dimensional Analysis," in Emotions: Essays on Emotion Theory, Stephanie Van Goozen, Anne E. Van de Poll, and Joseph A. Sergeant, eds., Hillsdale, NJ: Lawrence Erlbaum, 97-134.

—, Bruce N. Cuthbert, and Peter J. Lang (1991), "Startle and Emotion: Lateral Accoustic Probes and the Bilateral Blink," Psychophysiology, 28 (3), 285-295.

— Neuroscience of Emotion, Richard D. Lane and L. Nadel, eds., New York: Oxford University Press, 242-276.

Cacioppo, John T, and Wendi L. Gardner (1999), "Emotion," Annual Review of Psychology, 50, 191-214.

— Components: Form Follows Function," Journal of Personality and Social Psychology, 76,839-855.

Cook, Edwin W, III (2003), VPM Reference Manual, Birmingham, AL: author.

Dawson, Michael E., Ann M. Schell, and Dianne L. Fillion (2000), "The Electrodermal System," in Handbook of Psychophysiology, 2nd ed., John T Cacioppo and Louis G. Tassinary, eds., New York: Cambridge University Press, 200-223.

Dimburg, Ulf (1990), "Facial Electromyographic Reactions to Autonomie Activity to Auditory Stimuli," Biological Psychology, 31 (2), 137-147. 
Ekman, Paul (1993), "Facial Expression and Emotion," American Psychologist, 48 (4), 384-392.

Filopowicz, Allan (2000), "The Effect of Humor on Individual and Group Task Performance," Ph.D. dissertation, Harvard University.

Gorn, Gerald, Michel Pham Tuan, and Leo Yatming Sin (2001), "When Arousal Influences Ad Evaluation and Valence Does Not (and Vice Versa)," Journal of Consumer Psychology, 11 (1), 43-55.

Grabe, Margaret E., Annie Lang, Shuhua Zhou, and Paul D. Bolls (2000), "Cognitive Access to Negatively Arousing News: An Experimental Investigation of the Knowledge Gap," Communication Research, 27 (1), 3-26.

Gunter, Barrie (2002), Media Sex, Mahwah, NJ: Lawrence Erlbaum.

Hazlett, Richard L., and Sasha Y Hazlett (1999), "Emotional Response to Television Commercials: Facial EMG Vs. Self Report, " Journal of Advertising Research, 39 (2), 7-23.

Henthorne, Tony L., Michael S. LaTour, and Rajan Nataraajan (1993), "Fear Appeals in Print Advertising: An Analysis of Arousal and Ad Response," Journal of Advertising, 22 (2), 56-69.

Hopkins, Robert, and James E. Fletcher (1994), "Electrodermal Measurement: Particularly Effective for Forecasting Message Influence on Sales Appeal," in Measuring Psychological Responses to Media, Annie Lang, ed., Hillsdale, NJ: Lawrence Erlbaum, 113-132.

Ito, Tiffany A., and John T. Cacioppo (2000), "Electrophysiological Evidence of Explicit and Implicit Categorization Process," Journal of Experimental Social Psychology, 36 (6), 660-676.

Jancke, Lutz, Joachim Vogt, Musial Frauke, Lutz Kai, and Karl T. Kalveram (1996), "Facial EMG Responses to Auditory Stimuli," International Journal of Psychophysiology, 22 (1-2), 85-96.

Jansen, Dorine M., and Nico H. Frijda (1994), "Modulation of the Acoustic Startle Response by FilmInduced Fear and Sexual Arousal," Psychophysiology, 31 (5), 565-571.

Klimesch, Wolfgang (1994), The Structure of Long-Term Memory: A Connectivity Model of Semantic Processing, Hillsdale, NJ: Lawrence Erlbaum.

Lang, Annie (2000), "The Limited Capacity Model of Mediated Message Processing," Journal of Communication, 50 (1), 46-71.

(2006), "Motivated Cognition (LC4MP): The Influence of Appetitive and Aversive Activation on the Processing of Video Games," in Digital Media: Transformation in Human Communication, P. Messaris and L. Humphries, eds., New York: Peter Lang, 237-256.

, Paul D. Bolls, Robert F. Potter, and Karlynn Kawahara (1999), "The Effects of Production Pace and Arousing Content on the Information Processing of Television Messages," Journal of Broadcasting and Electronic Media, 43 (4), 451-475. 
— John Newhagen, and Byron Reeves (1996), "Negative Video as Structure: Emotion, Attention, Capacity, and Memory," Journal of Broadcasting and Electronic Media, 40 (4), 460-477.

-, Mija Shin, and Seungwhan Lee (2005), "Sensation Seeking, Motivation, and Substance Use: A Dual System Approach," Media Psychology, 1 (1), 1-29.

_ Kevin Wise, Seungwhan Lee, and Xiaolei Cai (2003), "Cognitive, Emotional, and Attitudinal Responses for Product and Alcohol Billboard Advertising," in Sex and Advertising, Tom Reichert and Jacqueline Lambias, eds., Mahwah, NJ: Lawrence Erlbaum, 107-132.

Lang, Peter (1979), "A Bio-informational Theory of Emotional Imagery," Psychophysiology, 16 (6), 495512.

—, Mark K. Greenwald, Margaret M. Bradley, and Alfons Hamm (1993), "Looking at Pictures: Evaluative, Facial, Visceral, and Behavioral Responses," Psychophysiology, 30 (3), 261-273. Larsen, Jeff T, A. Peter McGraw, and John T. Cacioppo (2001), "Can People Feel Happy and Sad at the Same Time?" Journal of Personality and Social Psychology, 81 (4), 684-696.

, Catherine J. Norris, and John T Cacioppo (2003), "Effects of Positive and Negative Affect on Electromyographic Activity over Zygomaticus Major and Corrugator Supercilii," Psychophysiology, 40 (5), 776-787.

LaTour, Michael S. (1990), "Female Nudity in Print Advertising: An Analysis of Gender Differences in Arousal and Ad Response," Psychology and Marketing, 1 (1), 65-81.

- and Robert E. Pitts (1989), "Using Fear Appeals in Advertising for AIDS Prevention in the College-Age Population," Journal of Health Care Marketing, 9 (September), 5-14.

—, and Herb Rotfeld (1997), "There Are Threats and (Maybe) Fear: Theory and Confusions of Appeals to Fear and Fear Itself" Journal of Advertising, 26 (3), 45-59.

—, and John F. Tanner, Jr. (2003), "Radon: Appealing to Our Fears," Psychology and Marketing, 20 (5), 377-394.

Lee, Sungkyoung, and Robert F. Potter (2005), "Do Words Matter? Effects of Emotional Words in Radio Messages on Orienting, Physiological Arousal, and Facial EMG Response," paper presented at the annual conference of the International Communications Association, New York, May.

Mandese, Joe (2005), "Viewing the Viewers: New Consumer Research Moves Closer to Behavioral Targeting," Broadcasting and Cable (August 22), 12-13.

Miller, Neal E. (1961), "Some Recent Studies on Conflict Behavior and Drugs," American Psychologist, 16, $12-24$. 
Murry, John P., and Peter A. Dacin (1996), "Cognitive Mediators of Negative-Emotion Effects: Implications for Understanding Media Context," Journal of Consumer Research, 22 (2), 439-447.

Osgood, Charles, George Suci, and Percy Tannenbaum (1957), The Measurement of Meaning, Urbana: University of Illinois Press.

Puccinelli, Nancy M. (1999), "Errors in Mood Regulation: Do We Know What's Best for Us?" Ph.D. dissertation, Harvard University. Potter, Robert F. (2000), "The Effects of Voice Changes on Orienting and Immediate Cognitive Overload in Radio Listeners," Media Psychology, 2 (2), 147177.

_ and Jimnyung Choi (in press), "The Effects of Auditory Structural Complexity on Attitudes, Attention, Arousal and Memory," Media Psychology.

Reeves, Byron, and Clifford Nass (1996), The Media Equation, New York: Cambridge University Press. Reichert, Tom (2002), "Sex in Advertising Research: A Review of Content, Effects, and Functions of Sexual Information in Consumer Advertising," Annual Review of Sex Research, 13,241-273. Shapiro, Stewart, Deborah J. MacInnis, and C. Whan Park (2002), "Understanding Program-Induced Mood Effects: Decoupling Arousal from Valence," Journal of Advertising, 31(4), 15-26.

Stern, Robert M., William J. Ray, and Karen S. Quigley (2001), Psychophysiological Recording, 2nd ed., New York: Oxford University Press.

Tassinary, Louis G., and John T Cacioppo (2000), "The Skeleto-motor System: Surface Electromyography," in Handbook of Psychophysiology, John T Cacioppo, Louis G. Tassinary, and Gary G. Berntson, eds., New York: Cambridge University Press, 200-223.

Thayer, Robert E. (1967), "Measures of Activation Through Self Report," Psychological Reports, 20 (2), 663-678.

$\longrightarrow$ (1989), The Biophysiology of Mood and Arousal, New York: Oxford University Press.

Wexler, Bruce E., Stephen Warrenburg, Gary E. Schwartz, and Larry D. Janer (1992), "EEG and EMG Responses to Emotion-Evoking Stimuli Processed Without Conscious Awareness," Neuropsychologia, 30 (12), 1065-1079.

Witte, Kim (1992), "Putting the Fear Back into Fear Appeals: The Extended Parallel Process Model," Communication Mono graphs, 59 (December), 329-349. 